\title{
Management of Non-Traumatic Acute Abdominal Pain in Children: The Couple Standard Radiography/Ultrasound, Is It Still Relevant?
}

\author{
Atafy Gaudens Dieth', N'goran Kouamé ${ }^{2 *}$, Abdoulramane Soumma ${ }^{2}$, Fotso Sorel Manewa ${ }^{2}$, \\ Anne-Marie Ngoan-Domoua ${ }^{2}$, Roger Daniel Ngbesso ${ }^{2}$ \\ ${ }^{1}$ Service de chirurgie pédiatrique et viscérale, $\mathrm{CHU}$ de Yopougon, Abidjan, Ivory Coast \\ ${ }^{2}$ Service de radiologie, $\mathrm{CHU}$ de Yopougon, Abidjan, Ivory Coast \\ Email: "kngoran@yahoo.fr
}

Received 20 February 2016; accepted 5 March 2016; published 9 March 2016

Copyright (C) 2016 by authors and OALib.

This work is licensed under the Creative Commons Attribution International License (CC BY).

http://creativecommons.org/licenses/by/4.0/

(c) (i) Open Access

\section{Abstract}

Objective: To evaluate the contribution of the standard radiography-ultrasound couple in the assessment of nontraumatic acute abdominal pain in children. Patient and Method: A retrospective study was carried out on a period of 2 years in the departments of radiology and pediatric surgery at the university hospital of Yopougon (Abidjan, Ivory Coast). It concerned 93 children hospitalized with acute nontraumatic abdominal pain in whom it was performed a laparotomy after a radiological diagnosis. Results: The mean age was 6.8 years old and the sex ratio was 1.5. The diffuse abdominal pain was the most common ground for admission $(43.3 \%)$ and abdominal ultrasonography was the most requested examination (51.6\%). The objectified etiologies were respectively appendicitis $(32.2 \%)$, peritonitis $(27.9 \%)$, the acute intussusception $(13.9 \%)$, occlusion $(12.9 \%)$, hypertrophic stenosis pylorus $(\mathbf{7 . 5 \%})$, cholecystitis $(3.2 \%)$ and the mesenteric adenolymphitis $(3.2 \%)$. The positive predictive value of radiological findings was greater than $85 \%$. Conclusion: The standard radiography-ultrasound couple remains essential in the treatment of nontraumatic abdominal pain of the child with a positive predictive value of $85 \%$.

\section{Keywords}

Acute Nontraumatic Abdominal Pain, Ultrasound, Standard Radiography, Pediatric Surgery, Child Abdominal Pain, Saharan Africa

Subject Areas: Pediatrics

${ }^{*}$ Corresponding author.

How to cite this paper: Dieth, A.G., Kouamé, N., Soumma, A., Manewa, F.S., Ngoan-Domoua, A.-M. and Ngbesso, R.D. (2016) Management of Non-Traumatic Acute Abdominal Pain in Children: The Couple Standard Radiography/Ultrasound, Is It Still Relevant? Open Access Library Journal, 3: e2084. http://dx.doi.org/10.4236/oalib.1102084 


\section{Introduction}

Les douleurs abdominales aiguës constituent une cause fréquente de consultation aux urgences. Aux États-Unis elles représentaient $6 \%$ des accès aux urgences pédiatriques de 1998 à 2008 [1]. En fonction de l'âge de l'enfant, les étiologies des douleurs abdominales diffèrent, pouvant être en rapport avec des causes banales ou, au contraire, nécessiter une prise en charge chirurgicale urgente [2]. L'interrogatoire, les signes associés, l'examen clinique et le bilan biologique orientent la démarche diagnostique, mais l'imagerie est souvent nécessaire pour le diagnostic de certitude. On dispose de nombreux moyens d'imagerie dont certains sont irradiants notamment la radiographie conventionnelle et la tomodensitométrie (TDM) et d'autres non irradiants: l'échographie et l'imagerie par résonance magnétique (IRM). Les méthodes non irradiantes sont à privilégier chez l'enfant par rapport aux méthodes utilisant les rayons $\mathrm{X}$ à cause du terrain pédiatrique qui est un terrain en pleine croissance et par conséquent sensible aux effets cancérigènes des rayons $X$.

Du fait de l'indisponibilité de et du coût élevé de l'IRM et de la TDM, l'échographie et le cliché de l'abdomen sans préparation restent les seules méthodes d'exploration des douleurs abdominales aiguës de l'enfant en Afrique subsaharienne. D'où l'intérêt de s'interroger sur l'apport réel du couple radiographie standard-Echographie dans la prise en charge des douleurs abdominales aigues non traumatique de l'enfant à l'heure de l'évolution des techniques d'imagerie médicale.

\section{Patients et Methodes}

Il s'agit d'une étude rétrospective descriptive qui s'est déroulée dans le centre hospitalier universitaire de Yopougon dans les services de radiologie et de chirurgie viscérale pédiatrique. La période d'étude était de 02 ans allant du 01 janvier 2013 au 31 décembre 2014. Elle a concerné 93 enfants hospitalisés pour douleur abdominale aigue non traumatique chez qui il a été effectué une laparotomie après la réalisation d'examens radiologiques. Ces différents examens radiologiques étaient l'échographie abdominale et la radiographie standard (cliché de l'Abdomen sans préparation-ASP-, la radiographie thoracique). L'échographie abdominale utilisait le plus souvent le mode B à l'aide de 2 sondes (convexe 3 - 5 Mhz et linéaire 7 - 10 Mhz). Il y’avait pas eu de cas de TDM, d'IRM ni d'opacifications digestives.

Les paramètres étudiés étaient l'âge, le sexe, le motif d’admission, les examens radiologiques réalisés, les étiologies objectivés à la radiologie et celles retenues après la laparotomie.

Les données ont été recueillies à partir des dossiers médicaux, des registres d'hospitalisation et des comptes rendus opératoires. Le traitement de ces données a été effectué à partir du logiciel SPSS version 16.0.

\section{Resultats}

Les différents résultats sont détaillés dans les Tableaux 1-3.

L'âge moyen de nos patients était de 6.8 ans avec une prédominance de la tranche d'âge entre 11 et 15 ans et des extrêmes allant de 02 jours à 15 ans. Il existait une prédominance masculine avec un sex- ratio égal à 1.5. La douleur abdominale diffuse était le motif le plus fréquent d'admission soit $43 \%$, suivi de celle de la distension abdominale $25.8 \%$. L'échographie abdominale a été l'examen le plus demandé avec un taux de $51.6 \%$. Chez 27 patients (29\%), il a été réalisé à la fois l'ASP et l'échographie abdominale. Les étiologies objectivées étaient respectivement l'appendicite (32.2\%), la péritonite (27.9\%), l'invagination intestinale aigue (13.9\%), l'occlusion (12.9\%), la sténose hypertrophique du pylore (7.5\%), la cholécystite (3.2\%) et l'adénolymphite mésentérique (3.2\%). La valeur prédictive positive des résultats radiologiques était de $85.7 \%$.

\section{Tableau 1. Moyens d'imagerie utilisée.}

\begin{tabular}{ccc}
\hline Examen & Fréquence & Pourcentage \\
\hline Echographie & 62 & $51.6 \%$ \\
ASP & 56 & $46.6 \%$ \\
Radio du thorax & 4 & $1.6 \%$ \\
Total & $\mathbf{1 2 0}$ & $\mathbf{1 0 0}$ \\
\hline
\end{tabular}

L’échographie a été l'examen le plus demandé avec un taux de 51.6\%. Chez 27 patients, il a été à la fois l'ASP et l’Echographie. 
Tableau 2. Pathologies diagnostiquées.

\begin{tabular}{ccc}
\hline Résultat & Fréquence & Pourcentage \\
\hline Appendicite & 30 & 32.2 \\
Péritonite & 26 & 27.9 \\
Invagination intestinale & 13 & 13.9 \\
Occlusion & 12 & 12.9 \\
Sténose hypertrophique & 7 & 7.5 \\
Cholécystite & 3 & 3.2 \\
Adénolymphite mésentérique & 2 & 2.1 \\
Total & $\mathbf{9 3}$ & $\mathbf{1 0 0}$ \\
\hline
\end{tabular}

L’appendicite et la péritonite étaient les pathologies les plus représentées avec un taux de 32.3\% et 28\%.

Tableau 3. Concordances diagnostiques entre la radiologie et la chirurgie.

\begin{tabular}{|c|c|c|c|}
\hline Pathologies & Chirurgie & Radiologie & VPP (\%) \\
\hline Appendicite & 33 & 30 & 91 \\
\hline Péritonite & 27 & 26 & 96.2 \\
\hline Invagination & 13 & 13 & 100 \\
\hline Occlusion & 14 & 12 & 85.7 \\
\hline Sténose hypertrophique de pylore & 7 & 7 & 100 \\
\hline
\end{tabular}

La valeur prédictive positive (VPP) des résultats radiologiques est très élevée (Supérieure à 85\%).

\section{Discussion}

Dans notre étude, l'âge moyen des enfants était de 6.8 ans et des extrêmes allant de 02 jours à 15 ans. La tranche d’âge de 11 à15 ans était la plus fréquente avec une proportion de $31 \%$. Nos résultats concordent avec ceux obtenus dans les études de Mabiala-Babela et col [3] au Congo qui a objectivé un âge de 6,5ans. Il existe par contre une discordance avec ceux d'AUREL et col [4] en France qui ont trouvé un âge moyen inférieur à 5 ans. Cette différence pourrait s'expliquer non seulement par la race voire le niveau de développement mais également le fait que leur étude englobe les douleurs traumatiques, non traumatiques et d'étiologie médicale. Tandis que nous avons pris en compte uniquement les douleurs non traumatiques d'étiologie chirurgicale.

Les enfants de sexe masculin sont les plus concernés par les douleurs abdominales non traumatiques. Dans notre étude nous avons mis en évidence un sex-ratio de 1.5 soit $59 \%$ des hommes contre $41 \%$ de sexe féminin. Nos résultats concordent avec les données de la littérature [3]-[5].

Dans notre série nous avons retrouvé que la douleur abdominale diffuse dominait le tableau des motifs d'admission dans une proportion avoisinant la moitié des cas (43\%) suivie de la distension abdominale qui est de 25.8\%. Nos résultats concordent avec les données de la littérature notamment avec l'étude d'Aurel et col [4] en France dans laquelle les douleurs abdominales diffuses représentent 35\%.

L'exploration des douleurs abdominales bénéficie de l'apport incontournable de l'imagerie médicale. Il s'agit d'un motif fréquent à la fois d'admission à la fois aux urgences pédiatrique et en hospitalisation. Mais il s'agit surtout de motif de demande d'examen d'imagerie médicale. Dans notre série nous avons trouvé que la douleur abdominale diffuse dominait les motifs d'admission soit $43 \%$ suivie de la distension abdominale qui était de 25.8\%. Nos résultats sont concordants avec les données de la littérature [3]-[5]. L’échographie a été l'examen le plus sollicité dans notre étude (51.6\%) dans un contexte de plateau technique pauvre où il n'y a pas d'IRM et où la TDM est indisponible. Certainement du fait de son innocuité, sa reproductibilité et la rapidité à son accès. Particulièrement performante chez l'enfant, elle présente également l'avantage d'apprécier le péristaltisme, la compressibilité et la vascularisation digestive. Ces atouts compensent largement son caractère opérateur dépen- 
dant [6] [7]. Les examens irradiants étaient représentés par l'abdomen sans préparation (46.6\%). Le couple échographie et abdomen sans préparation a été réalisé dans environ un tiers des cas. Nous avons réalisé 4 radiographies thoraciques qui ont objectivé un croissant gazeux (pneumopéritoine). Ces différents examens d'imagerie nous ont permis de mettre en évidence les pathologies à l'origine de la douleur abdominale non traumatique (Figure 1 et Figure 2) dominées par l'appendicite aigue (32.2\%) suivie de la péritonite (27.9\%), invagination intestinale aigue (13.9\%) et les occlusions intestinales (12.9\%).

Ces résultats témoignent de l'importance de l'échographie dans le diagnostic des étiologies de douleurs abdominales aigues non traumatiques de l'enfant et sont conformes avec les données de la littérature [7]-[9]. Cependant dans notre étude un cas d'invagination intestinale aigue et d'appendicite ont été diagnostiqués uniquement sur le cliché de l'abdomen sans préparation d'où la nécessité de maitriser certains signes clés de cet examen (Figure 1 et Figure 2). La pertinence des résultats radiologiques a été évaluée après la laparotomie. Ce qui nous a permis de calculer la valeur prédictive positive (VPP) des résultats radiologiques qui était très élevée : supérieure à $85 \%$ pour la radiographie et de $100 \%$ pour l'échographie. Ce résultat est concordant avec celui d'autres auteurs qui s'accordent sur l'importance primordiale de l'échographie dans le diagnostic des douleurs abdominales aigues non traumatiques de l'enfant. MOBIMA [10] a trouvé une valeur prédictive positive de $90 \%$.

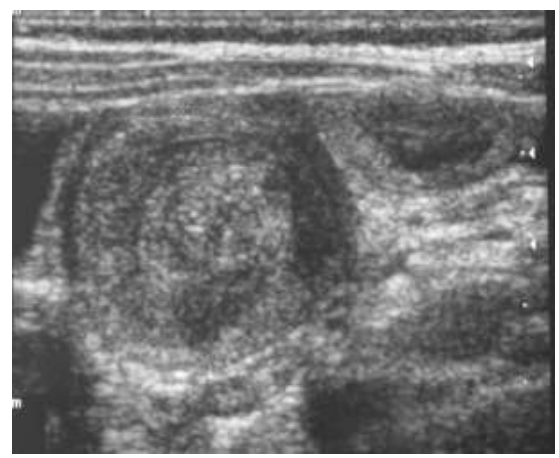

(a)

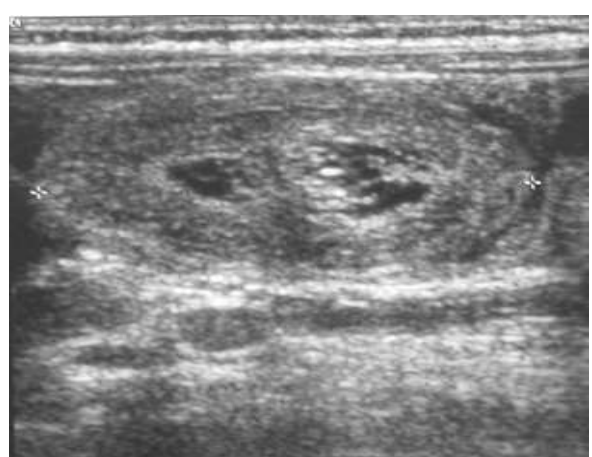

(b)

Figure 1. Echographie abdominale à l'aide d'une sonde de haute fréquence: Image en cible en coupe transversale (a) et en pseudo-rein en coupe longitudinale (b): Invagination intestinale aigue.

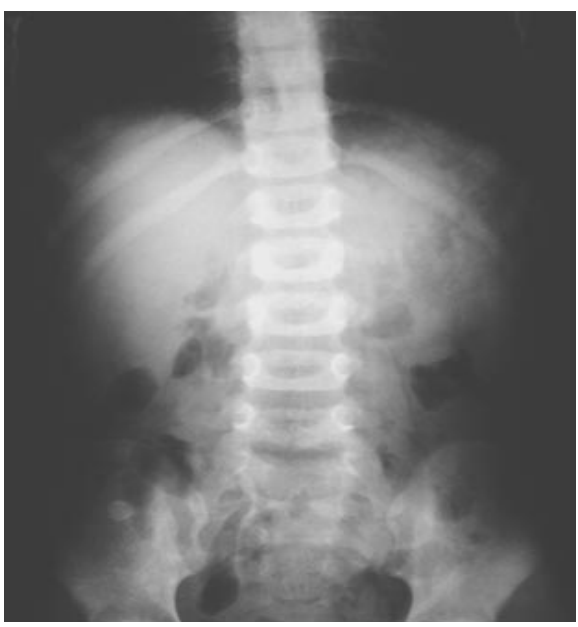

(a)

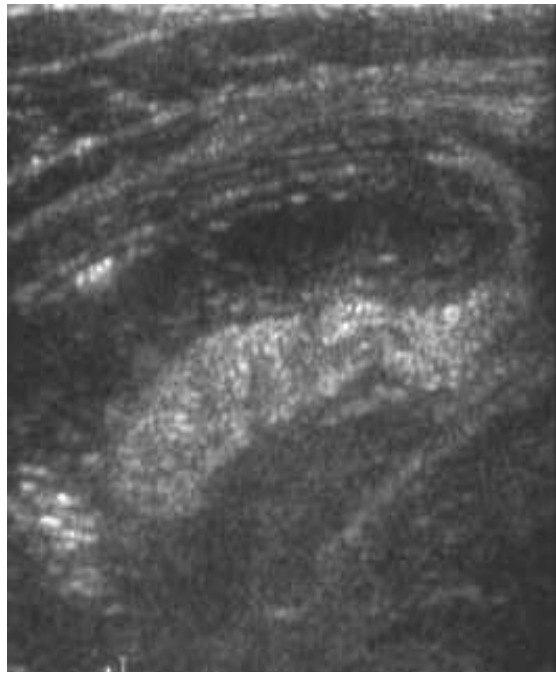

(b)

Figure 2. Enfant âgé de 15 ans, de sexe masculin, présentant une douleur péri ombilicale, avec contracture abdominale et fièvre $\left(39^{\circ} \mathrm{C}\right)$. (a): inclinaison latérale du rachis et une stercolithe à l’ASP (flèche). (b): inflammation appendiculaire à l'échographie. 


\section{Conclusion}

L’imagerie médicale en Afrique subsaharienne, malgré la pauvreté du plateau technique demeure incontournable dans la pris en charge des douleurs abdominales aiguës non traumatiques de l'enfant. L'échographie couplée à l'abdomen sans préparation et parfois à la radiographie du thorax permettent de faire le diagnostic positif avec une valeur prédictive positive très élevée. Afin d’en tirer tous les bénéfices, la prise en charge des douleurs abdominales aigues non traumatiques de l'enfant doit associer les radiologues et les chirurgiens pédiatres dans le cadre d'une réunion de concertation pluridisciplinaire.

\section{Conflits d'Interet}

Aucun.

\section{References}

[1] Fahimi, J., Herring, A., Harries, A., Ralph Gonzales, R. and Alter, H. (2012) Computed Tomography Use among Children Presenting to Emergency Departments with Abdominal Pain. Pediatrics, 130, 1069-1075. http://dx.doi.org/10.1542/peds.2012-0739

[2] McCollough, M. and Sharieff, G.Q. (2006) Abdominal Pain in Children. Pediatric Clinics of North America, 53, 107137. http://dx.doi.org/10.1016/j.pcl.2005.09.009

[3] Mabiala-Babela, J.R., Pandzou, N., Koutaba, E., Ganga-Zandzou, S. and Senga, P. (2006) Etude rétrospective des urgences chirurgicales viscérales de l'enfant au chu de Brazzaville (Congo). Médecine tropicale, 66, 172-176.

[4] Aurel, M. (2007) Douleurs abdominales aigues non traumatiques de l'enfant. Masson, Paris, 435 p.

[5] Lougue-Sorgho, L.C. (2005) Quelles méthodes d'imageries dans l'exploration d'une douleur abdominale de l'enfant à Ouagadougou? Journal de Radiologie, 86, 223-233.

[6] Ramirez, R., Chaumoitre, K., Michel, F., Sabiani, F. and Merrot, T. (2009) Occlusion intestinale de l'enfant par mal rotation intestinale isolée. À propos de 11 cas. Archives de pÉdiatrie, 16, 99-105. http://dx.doi.org/10.1016/j.arcped.2008.11.010

[7] Vignon, P. and Cholley, B. (2005) Échographie portable chez les patients en état critique. Réanimation, 14, $692-699$. http://dx.doi.org/10.1016/j.reaurg.2005.10.018

[8] Marret, H. and Tranquart, F. (2010) Échographie et IRM pelviennes, ou la surenchère est-elle justifiée? Gynécologie Obstétrique Biologie \& Fertilité, 38, 163-165. http://dx.doi.org/10.1016/j.gyobfe.2010.01.001

[9] Lambot, K., Gorincour, G., Le Hors, H. and Chapuy, S. (2006) Douleur abdominale aigue non traumatique de l'enfant: Apport respectif de l’ASP et de l'echographie. Journal de Radiologie, 87, 1322. http://dx.doi.org/10.1016/S0221-0363(06)87117-9

[10] Vandertum, L., Vunda, A., Gehri, M., Schanze, O., Hanquinet, S. and Gervaix, A. (2011) Invagination intestinale chez l'enfant : une triade vraiment classique. Revue médicale suisse, 7, 451-455. 